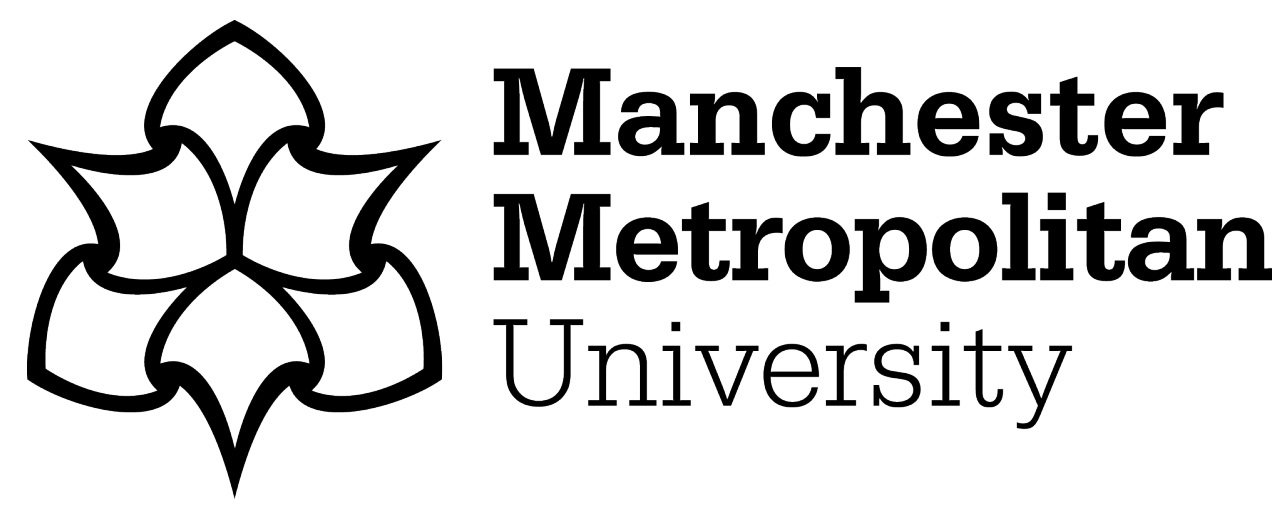

Sustere, Edite and Tarpey, Emma ORCID logoORCID: https://orcid.org/00000003-2238-2719 (2019) Least restrictive practice: its role in patient independence and recovery. The Journal of Forensic Psychiatry \& Psychology, 30 (4). pp. 614-629. ISSN 1478-9949

Downloaded from: https://e-space.mmu.ac.uk/625233/

Version: Accepted Version

Publisher: Taylor \& Francis

DOI: https://doi.org/10.1080/14789949.2019.1566489

Please cite the published version 


\section{Least Restrictive Practice: Its Role in Patient Independence}

\section{and Recovery.}

Edite Sustere $^{\mathrm{a} *}$ and Emma Tarpey

${ }^{a}$ Humber Teaching NHS Foundation Trust, Beverley Road, Willerby Hill, Willerby, England, HU106ED, edite.sustere@nhs.net; ${ }^{b}$ Manchester Metropolitan University, Brooks Building, 53 Bonsall Street, Manchester, England, M15 6GX

Acknowledgment: We'd like to thank Humber Teaching NHS Foundation Trust for allowing us to carry out the research.

Disclosure statement: At the time of the research, ES was employed by Humber Teaching NHS Foundation Trust. No conflict of interest declared by ET.

Funding details: No funding was sought for the following research project. 


\section{Least Restrictive Practice: Its Role in Patient Independence and Recovery.}

One of the five overarching principles of the Mental Health Act: Code of Practice (2015) is to provide patients with care and treatment which is least restrictive whilst encouraging recovery and promoting independence. However, there is limited research which explores the application of these principles within a medium secure unit. The aims of the research were to explore what are patient's experiences of least restrictive practices and to what extent do they perceive that least restrictive practices maximise their independence and recovery. Semistructured interviews were carried out with twelve male inpatients within a medium secure unit. Five themes were evident: Positive Changes, Perceived Lack of Transparency, Social Isolation, Institutionalisation and Normality. It was found that patient's perceived that there was lack of shared understanding between staff and patients of what is considered least restrictive. Patient recovery was promoted through positive risk taking, the reduction in the use of seclusion and through promotion of meaningful activities that resembled a life in the community. Nevertheless, patients perceived that there was lack of opportunities to socialise with patients from other wards. Due to the security level of the hospital patients perceived that independence was not achievable.

Keywords: least restrictive practice, forensic mental health, secure settings, mental health act, recovery

Word count: 4829 


\section{Introduction}

Individuals with neuro-developmental or mental disorders who may be at risk of harm to themselves or others, and whose risk of harm may not be safely managed within a home, community or hospital setting might require treatment in secure mental health settings (Department of Health, 2015). In such settings, relational, physical and procedural security measures are implemented to ensure safe delivery of care whilst effectively managing risk (Mezey, Kavuma, Turton, Demetriou \& Wright, 2010). Least restrictive refers to the process whereby physical, relational and procedural restrictions are kept to a minimum and only implemented when necessary (Kennedy, 2002). The Mental Health Act: Code of Practice states that least restrictive practices (LRPs) should maximise patient independence and promote recovery. However, there is lack of literature exploring the impact of LRPs on patient independence and recovery.

The impact of LRPs on patient independence and recovery are important points of exploration as coercive and restrictive ward practices are the biggest predictors of violence (Whittington \& Richter, 2006). Enforcement of restrictive practices coupled with lack of understanding of patient's experiences may result in power struggles (Alexander \& Bowers, 2004). Often patient-staff conflicts within forensic services are due to what they perceive as unfair restrictions (Whittington \& Richter, 2006) and highly restrictive environments can contribute towards tensions on the ward (Meehan, McIntosh \& Bergen, 2006). Therefore, it is crucial to consider service user perspectives to help develop and implement LRPs (Atkinson, 2002). Some of the key elements to recovery are having meaning, hope and purpose (Drennan \& Aldred, 2012). However, living in a secure hospital, where patients are required to live within a very structured and compulsory environment, recovery might be difficult to achieve (Mezey et al., 2010). Individuals are likely to have histories of multiple traumas, complicated emotional and interpersonal needs as well unmet criminogenic needs (Mann, Matias \& 
Allen, 2014) therefore, nurses may subconsciously create restrictions to help manage complex situations and clients (Finnema, Louwerens, Slooff \& van-den-Bosch, 1996) therefore finding a balance between recovery and risk management can be difficult (Drennan \& Aldred 2012).

LRPs should also maximise patient independence (Department of Health, 2015) which is an important component of recovery in mental health along with self-direction, and ability to determine your own journey of recovery (Alexander \& Bowers, 2004). Restrictions on patient independence within forensic services make recovery difficult, and also maintain feelings of powerlessness and hopelessness (Livingston, Rossiter \& Verdun-Jones, 2011). Staff members should therefore facilitate patients understanding of the rationale behind the restrictions, and apply a flexible and individualised approach when imposing ward restrictions (Alexander, 2006). However, professionals might be unsure how to promote healthy independence whilst managing risk (Jamieson, Taylor \& Gibson, 2006). In Jamieson's et al. (2006) study professionals acknowledged that secure services are created as a place for dependency. This is because these services provide safety, absence from drugs, therapy and support.

Overall, much of the focus within current literature has been on reducing the risk of re-offending and little attention has been paid on how ward rules can increase patient independence and promote recovery (Urheim, Rypdal, Palmstierna, \& Mykletun, 2011). The Good Lives Model (GLM) (Ward \& Brown, 2004) was chosen to interpret the results as it focuses on paying attention to the individual's strengths and protective factors. By focusing on strengths and risk factors together will create a shared understanding between the patient and their team of their risks but also safety (Barnao, Robertson \& Ward, 2010). Therefore, whilst restrictions are still in place, these are being kept to a minimum and a strength-focused approach is adapted. It has also shown 
to improve engagement in treatment and reduce drop-out rates (Barnao et al., 2010). The GLM model suggests that all human action, including offending, is considered to be a way of seeking primary human goods (PHGs) (Ward \& Brown, 2004). Research suggests that there are eleven PHGs: life; knowledge; excellence in play; excellence in work; agency; inner peace; relatedness; community; spirituality and happiness (Ward \& Brown, 2004). There is a commonality of PHGs; however, the means of achieving them may be flawed by process of hospitalisation (Ward \& Brown, 2004). GLM is used to support individuals to have 'a good life' without re-offending, and to provide purpose and meaning. The following study aims to apply to the GLM model to review how the PHGs are achieved within a medium secure service to achieve independence and recovery using LRPs.

Therefore, this research aims to explore:

- What is patients understanding of LRPs?

- To what extent do patients perceive that LRPs promote their recovery?

- To what extent do patients perceive that LRPs maximise their independence?

\section{Method}

\section{Design}

A qualitative research design was employed. The epistemological position of this research is that of realist. Patients' experiences were theorised in a straight forward way, because the information provided by the participants would allow the researcher to articulate experience and meaning (Braun \& Clarke, 2013). The researcher wasn't trying to theorise any socio-cultural contexts that may explain individual accounts but instead tried to capture issues that are important to the participants that the researcher may have not anticipated (Braun \& Clarke, 2013). It was also assumed that patient 
experiences of LRPs will change as they progress through their recovery especially as the concept of LRPs is relatively new. For example, key documents relating to LRPs date back to 2014 such as Positive and Proactive Care: Reducing the Need for Restrictive Interventions (2014) and Mental Health Act 1983: Code of Practice (2015).

\section{Participants}

An opportunity sampling technique was utilised. Posters were displayed on four medium secure wards and on one low secure ward which are all part of one National Health Service (NHS) hospital. Participants that volunteered for the study were provided with a Participant Information Sheet. 12 male participants over the age of 18 were recruited from four all-male medium secure wards. No participants from the low secure ward volunteered to participate which may have affected the results. A semistructured interview schedule was prepared using open-ended questions. Questions were used contextually and follow-up questions were asked to be responsive to the patient's developing account (Braun \& Clarke, 2013).

\section{Procedure}

All of the interested participants were assessed as having capacity to consent to participation by the Multi-Disciplinary Team (MDT) and the researcher. Patients were then approached by the researcher and invited for an interview. Interviews were audiorecorded to ensure that the richness of the data was captured. When each interview ended, recording was stopped and the researcher de-briefed each participant taking into account their presentation and wellbeing. 


\section{Analysis}

The epistemological position of this research was that of realist. This means that patient's experiences were theorised in a straight forward way, because it was assumed that the information provided by the participants would allow the researcher to articulate experience and meaning (Braun and Clarke, 2013). The data was analysed using guidelines produced by Braun et al. (2006). The themes were identified using an inductive approach. This meant that the themes were strongly related to the data and not driven by a specific pre-existing coding frame or theoretical interest. Instead, the analysis was data-driven to fully explore patient's experiences of LRP (Braun et al. 2006). The themes were analysed at a semantic level (Boyatzis, 1998). In this instance, codes were semantic features which were assessed as meaningful. Codes were identified and matched up with all the relevant data extracts to evidence those codes. The codes were then analysed to see how they may be combined and sorted into potential themes

\section{Ethics}

The research project was assessed and approved by the Health and Research Authority (HRA) and the local Research and Ethics Committee (REC).

\section{Results and Discussion}

Five themes were identified and interpreted using the GLM model:

\section{Theme 1: Positive Changes}

Several positive changes were observed by patients in regards to LRPs. Participants thought that staff supported patients to have more responsibility by engaging in positive 
risk taking. In addition, patients reported that seclusion was rarely used. These results are further explored in sub-themes Compassionate Care and Seclusion as Last Resort.

\section{Sub-theme 1: Compassionate Care}

Staff members were perceived as responsive to patient's needs and adopted a nonjudgmental approach when patients experienced crisis. When patients self-harmed staff helped patients to express how they felt rather than imposing restrictions through removal of risk items or seclusion.

“...like if you was self-harming...you get things locked up in your bedroom then you could lose it for a week...now they give me half an hour to calm me self down and give me some pen and paper and that way...I could draw how I feel...” (P1)

This individual was offered alternative support strategies to minimise their emotional stress, which is likely to help the patient develop more adaptive ways of coping.

"I mean every staff they do their hardest to go out of their way just to help patients... as soon as you actually see that the staff just wanna give you a hand it's great I mean we do need a hand sometimes...” (P2)

Participants spoke about the positive changes they noticed since the introduction and implementation of LRPs. The key to effective balance between treatment and security was the therapeutic relationship between staff and patients. In addition, positive risk taking had a significant impact on participants independence by promoting responsibility and personal choice. 
“...when I first come it was tight...it's like we was in the army or something...I prefer all the risks that they took with me...for me to have more responsibility..."(P4)

Sub-theme 2: Seclusion as Last Resort

Patients reported previously being restrained and taken to seclusion for minor incidents. However, patients reported that now seclusion was used as last resort when managing aggressive behaviour. Instead, early intervention and de-escalation strategies were utilised.

“...if you told a member of staff to fuck off or go away...they'd all bother ya knock you down and...make you go down to seclusion but now they don't take ya unless it's

$$
\text { necessary..." (P4) }
$$

“...the main thing really good in least restrictive that first option isn't to restrain somebody but in the past it always has been...when I first come here...you could get taken off the ward for swearing...” (P5)

\section{Theme 2: Perceived Lack of Transparency}

Patients perceived that there was lack of transparency between staff and patients regarding what is considered least restrictive. Similarly, patients weren't always given reasons behind denied requests and the outcomes of meetings. 
“...it's the front line staff who need to be education in what is considered to be least restrictive and what's not because...we don't know what we can and can't do... a lot of the time we haven't got a clue what's going on we rely on our staff to know what's happening...” (P6)

In this study, patients perceived that staff were not always aware of what is considered LRP. Patients reported that staff did not always communicate ward related LRPs to them. It is essential that staff members and patients are provided with appropriate support, education and guidance on LRPs. Furthermore, if a patient was told "no" in response to a request they weren't always provided reasons as to why their requests couldn't be completed.

“you're just told you can't you're not told why you can't... it's just petty can't do it somebody made a decision and you can't do it" (P7)

"depends on the person suppose who you've asked some staff might tell you why and be honest with you upfront and other staff might not..." (P8)

Similarly, patients reported that they weren't always kept up to date regarding developing policies and ward rules.

“...they seem to have meetings about meetings...you go to one person and they say oh that things gone to so and so and the next time you see them again ask oh patient council are dealing with it now...they don't always relate what's happened to other

$$
\text { people..." (P5) }
$$


Consequently, lack of information is unlikely to help patients become more independent and promote their recovery.

\section{Theme 3: Social Isolation}

Patients wished to have increased freedom of movement across the hospital. Although meeting at certain social events was helpful, it was recognised that there was still some isolation within the hospital.

“...one of the other restrictive practices has been a big thing about...keeping patients segregated so for example...if patients from [WARD] were at the shop and patients from [WARD] came down it was a big massive issue... by not letting patients mix nobody knows each other and it's all very difficult..." (P6)

“...you can't mix with them because they got learning disabilities you can't mix with them because they've got erm severe mental illness...it...stigmatises even in the place... they must feel you know how are we different to them...it must play on their minds" (P5)

Allowing patients to engage in activities that facilitate meeting new people is likely promote development of healthy peer relationships. However, social isolation was also observed on an individual ward. Talking in groups on corridors and walking down other patient's corridors was not allowed, although patients didn't understand why this was the case. 
“...talking in corridors...you're not allowed to yet we live together ...” (P7)

“...it's just a little place it's just depressing...if we go on the other side of the corridor they tell ya come back the other way...we should be allowed to go in each other's rooms...it's nice just to chill out in someone's room and just have a nice game” (P9)

\section{Theme 4: Institutionalisation}

Patients reported that independence was minimal within a medium secure unit.

“...you can't be independent when you're here...they've got a system they've got to stick to they've got the cleaner to clean up they've got the cooks to cook the food you've got the room you get to eat your food in you don't get independent” (P10)

"they do everything for us don't they you only do a few bits..." (P11)

Although some degree of control within forensic settings is inevitable, especially in the context of risk management, participants felt like they didn't have much control over the decisions made regarding risk management within the hospital. There was some evidence of hopelessness and just accepting that this is how things were. Consequently, this decreased patient's perceived independence. These perceptions were based on the security level of the hospital.

“...if you was asking me in another environment I'm sure the answers would be different but within this environment in terms of the level of security independence is minimum..." (P6) 
“...they've got to make it safe so they won't be able to make it independent will they”

\section{Theme 5: Normality}

LRPs helped patients to achieve a sense of normality when they were offered opportunities to engage in activities which reflected a life in the community.

“...I learnt to live by myself which was one of the bonuses of being in here and progressing and with the OT cooking sessions... which shows we are really getting prepared to go out in the community..." (P8)

Patients engaged in meaningful activities offered by the hospital which were likely to help them achieve the PHG of Happiness. For example, patients utilised community leave to engage in hobbies and activities which they found meaningful.

“...going to cinema...I've been like going to football matches and stuff like that which is good...least restrictive is good in that sense case giving people that freedom...to do normal things..." (P5)

Therefore, these are positive findings in terms of how LRPs have promoted and maximised patient independence and recovery. In addition, self-medication and having a bedroom key increased sense responsibility and control. 
“...now people can lock their own bedroom...just like if you had a key to your house...now majority of patients can actually give themselves their own medication people got control..." (P5)

Implementing practices that reflect a life in the community also further maximised participants recovery. Participants who were provided with opportunities to practice skills within a framework of LRPs, spoke much more positively of their idea of recovery and experiences of living within a secure environment.

"recovery means to me...to manage being able to manage your problems and living in the community without re-offending and so looking after yourself...” (P8)

Implementation of LRPs helped patients to develop those skills which are more likely to help patients feel prepared for discharge into the community to achieve a happy life.

\section{$\underline{\text { Discussion }}$}

This research aimed to explore patient's understanding of LRPs and whether LRPs promote patient independence and recovery. Five themes were identified 1. Positive Changes with sub-themes Compassionate Care and Seclusion as Last Resort, 2. Perceived Lack of Transparency, 3. Social Isolation, 4. Institutionalisation and 5. Normality.

The guidance for commissioners of forensic mental health services emphasises that "The application of security measures should aim to promote a safe and therapeutic environment, whilst pro-actively encouraging independence and recovery..." (Joint 
Commissioning Panel for Mental Health, 2013). However, there are lack of measures available which highlight whether independence and recovery are actually promoted. Therefore, the following study has applied the Good Lives Model to review what is patient understanding of LRPs and how primary human goods (primarily independence and recovery) are achieved within a medium secure service using the least restrictive approach.

The first theme of Positive Changes reflected the cultural change which had occurred since a greater focus was brought onto the principles of LRPs. Staff members were observed to present as compassionate and engage in positive risk taking, which promoted patient's sense of responsibility and independence. Receiving support and being able to make choices, are all important characteristics which positively affect treatment engagement, and likeliness to maintain engagement as demonstrated by the GLM (Mason et al., 2012). The GLM suggests that individuals have a desire for personal choice and independence. This is referred to as primary human good of Excellence in Agency (Ward and Brown, 2004). In this study, professionals were perceived as more understanding and non-judgmental whilst focusing on rehabilitation; possessing characteristics which promote patient independence, self-reliance and recovery (Alexander, 2006). Similarly, positive risk taking in mental health promotes patient's personal growth, autonomy and success (Robinson et al., 2007).

Second sub-theme Seclusion as Last Resort reflected the reduced use of seclusion. Seclusion is a controversial management strategy of aggressive and disrupted behaviour (Alexander, 2006). Patients reported that previously they could be transferred to seclusion for minor incidents such as swearing and arguing however, patients stated that 
now seclusion was last resort. Reduced seclusion is likely to increase patient inner peace (freedom from emotional turmoil and stress) as suggested by GLM. Inner Peace refers to individual's ability to have understanding and awareness of their emotions and how to achieve emotional balance through adaptive strategies (Ward and Brown, 2004). In this study, a patient was trying to achieve emotional turmoil through self-harming, and effective preventive interventions were actively applied, such as verbal deescalation and drawings as a way of expression emotion. Although seclusion may not always be avoidable, it is essential that it is done in a therapeutic manner by providing patients with a clear explanation for their seclusion, and are provided with enough opportunities to interact with professionals during their seclusion (Keski-Valkama, Koivisto, Eronen and Kaltiala-Heino, 2010). Indeed, early intervention, engaging the patients and evidence-based risk assessments are all positive strategies used to reduce violence and aggression (Maguire et al., 2012).

In contrast, Theme 2: Perceived Lack of Transparency demonstrated that patients were dependant on staff knowledge however, there was lack of understanding what is considered LRP. Detailed knowledge is essential to maintain a therapeutic alliance between staff and patients (Dimond et al., 2011). Rae (1993) argues that secure units apply a custodial culture in order to protect against staff anxiety and fear. This is maintained through lack of training and education of staff. If staff members feel confident in supporting patients, they are more likely to apply LRPs, rather than resulting to restrictive practices of just saying "no" without providing a reason (Maguire et al., 2012). Patients may feel sensitive, rejected, criticised and confused about the restriction, even when that wasn't the professional's intention (Alexander, 2006). Components that increase staff safety and confidence were colleague's knowledge, 
experience and ability. Staff welcome policies and training which ensures a skilful and experienced workforce (Martin and Daffern, 2006).

Poor information sharing may mean that patients are unable to achieve primary human good of Knowledge. Knowledge refers to individual's desire to understand themselves and their environment (Ward and Brown, 2004). Patients are more likely to be aggressive when they think that ward restrictions are imposed in a punitive manner (Alexander and Bowers, 2004). Genuinely engaging patients in the construction and evaluation of ward restrictions mat facilitate independence and improve patient-staff communication (Alexander, 2006).

Literature suggests that positive social relationships can have a significant impact on patient's recovery (Nijdam-Jones et al., 2015). Social cohesion has been the centre of government policies within mental health services, for example, No Health without Mental Health (DoH, 2011). However, in Theme 3: Social Isolation, patients perceived that there was little opportunity for creating positive, social relationships with their peers either on the ward, or generally across the hospital. Patients felt that certain restrictions on the ward, such as not going near other patient's bedrooms and lack of freedom of movement across the hospital meant there was little opportunity for social cohesion. This means that their primary goods of Friendship and Community are unmet. These primary human goods relate to the desire to establish bonds with others, and relate to being part of a group and being connected to others (Ward and Brown, 2004). These findings are consistent with previous literature where patients felt there were restrictions on their social environments, meaning that there was little opportunity for empowerment and recovery (Morris et al., 2016). If patients are restricted from 
achieving these goods through pro-social means, they're more likely to engage in unhelpful activities to seek belonginess elsewhere. Patients might form antisocial networks within hospital perimeters to achieve a sense of relatedness and community.

The Social Bonding Theory suggests that human morality is dependant to what extent an individual is able to bond with the society and others therefore, adopting society's morals (Hirschi, 2002). The development of healthy and pro-social relationships within the hospital may make the individual feel more socially equipped to develop healthy relationships once in the community. Relationships formed on psychiatric wards are likely to predict relationships on the outside, or their absence (Ward and Brown, 2004). Similarly, social cohesion and support from fellow patients are seen as essential foundations for effective rehabilitation (Bressington et al., 2011). Therefore, promoting social inclusion within a medium secure unit is likely to benefit patient's recovery, the services and society (CSIP, 2007).

Independence was perceived as a minimum within secure services which was reflected within Theme 4: Institutionalisation. There were certain procedures in place which, in the eyes of the patient, couldn't be changed because of the security level. Similar findings have been found by previous research, where the level of security and restrictions had a significant effect on patient's freedom and independence (Milsom et al., 2014). It is important to take into the account contextual factors which might affect the patient, such as ward practices and routines to avoid individuals feeling like they're being fitted into the hospital's existing processes (Barnao et al., 2015). According to GLM, by promoting patient's goals and supporting them with skills to achieve these goals are likely to restore independence (Barnao et al., 2015). However, an early onset 
of a mental illness can affect the development of certain skills, such as independent living. In addition, previously learnt skills may be temporarily affected (Barnao et al., 2010).

Although some degree of control is inevitable, especially in the context of risk management, it is important that clinicians remain mindful of the effects of this on the patient (Alexander, 2006). For example, some patients may just accept the ward rules which means they might be susceptible to institutionalisation. Patients might be afraid to express their feelings because this might be seen as a sign of deterioration in mental health (Alexander, 2006). Nevertheless, patients are not always included in the design, planning or delivery of forensic services (National Survivor User Network, 2011). It is essential that services involve patients as much as possible as they have direct experience and knowledge of being part of forensic services therefore, their expertise is invaluable

Patients reported that LRPs helped them achieve a sense of normality when offered opportunities to engage in activities which reflect a life in the community. For example, leave to the community helped patients to regain control and develop independence. Indeed, recovery can be much more than just about mental illness, it is about overcoming challenges, being able to work, study, love and live with a sense of meaning and purpose (Ward and Brown, 2004). According to GLM, some individuals use mental health services as a means of achieving their primary human goods until more normalised ways of achieving them can be used (Barnao et al., 2010). Indeed, the GLM encourages adapting treatment which will help patients learn skills which can be utilised to achieve their goals. However, if this not achieved, patients may result to 
controlling, dominating or abusing others to establish control (Ward and Brown, 2004). A focus on patient's strength's is likely to increase motivation and satisfaction of the ward (McMurran and Ward, 2004).

\section{Conclusion}

According to the participants there was still lack of shared understanding between staff and patients around what is considered LRP. Inconsistent responses, lack of communication and feelings of uncertainty behind some of the staff decisions affected participant's understanding of what are LRP within the hospital. Therefore, staff should work with patients to facilitate understanding of the reasons behind some of the restrictions, and involve patients in the decision making of the hospitals practices wherever possible.

Overall, patients perceived that positive risk taking, engaging in activities that reflect a life in the community and seclusion being used as last resort are all examples of how LRPs have had a positive impact on patient recovery.

Independence was seen as a minimum due to physical and procedural processes of the hospital rather than relational security. Therefore, it is important to remain mindful of the effects of risk management and avoid making patients feel like they're being 'fitted' into existing processes.

\section{Limitations}

A consultation with patients at the design and implementation stages of the research may have better represented patient's voices. This research was completed as part of a Master's degree qualification therefore one professional carried out the entire research. 
It is also possible that as the researcher works within the hospital it may have had an impact on the data. Participants may have felt hesitant to share their views about restrictive practices although there was a good balance of positive and negative views. The length of admission at the hospital was not recorded therefore, this was likely to have an influence on the results as patient experiences of LRPs are likely to change as they progress through their recovery. Patients at higher risk of violence may be subject to increase relational, procedural and physical security therefore, their perceptions of the ward may be different to other patients (de Vries, Brazil, Tonkin and Bullten, 2016). The research is explorative and the findings contribute to new understanding to the literature. However, not all medium secure units are identical. There may be differences in discharge pathways as well as differences in the therapeutic environment. Nevertheless, it is hoped that due to resemblances in the environments and similarities in treatments available the themes are applicable to other forensic settings. The findings only reflect patient's views and might not necessarily reflect the services practices although, these findings are consistent with previous research where entrenched practices within' closed institutions have been evident (Carr and Harvers, 2012). 


\section{References}

Alexander J., \& Bowers, L. (2004). Acute psychiatric ward rules: A review of the literature. Journal of Psychiatric and Mental Health Nursing, 11(5), 623-631.

Alexander, J. (2006). Patient's feelings about ward nursing regimes and involvement in rule construction. Journal of Psychiatric and Mental Health Nursing, 13(5), $542-553$.

Arif, Z., Barry, G., Duff, A., Hulatt, I., Foster, S., King, D., \& Parker, M. (2016). Positive and Proactive Care: Reducing the Need for Restrictive Interventions. London: Royal College of Nursing.

Atkinson, M.J. (2002). Least restrictive alternative - advance statements and the new mental health legislation. Psychiatric Bulletin, 26(7), 246-247.

Barnao, M., Robertson, P., \& Ward, T. (2010). Good Lives Model applied to a forensic population. Psychiatry, Psychology and Law, 17(2), 202-217.

Barnao, M., Ward, T., \& Robertson, P. (2015). The Good Lives Model: A new paradigm for forensic mental health. Psychiatry, Psychology and Law, 23(2), 288-301.

Bouman, Y. H. A., Schene, A. H., \& de Ruiter, C. (2009). Subjective well-being and recidivism in forensic psychiatric outpatients. International Journal of Forensic Mental Health, 8(4), 225-234.

Braun, V., \& Clarke, V. (2006). Using thematic analysis in psychology. Qualitative Research in Psychology, 3(2), 77-101. 
Braun, V., \& Clarke, V. (2013). Successful qualitative research: A practical guide for beginners. London: SAGE Publications Ltd.

Bressington, D., Stewart, B., Beer, D. and MacInnes, D. (2011). Levels of service user satisfaction in secure settings - A survey of the association between perceived social climate, perceived therapeutic relationship and satisfaction with forensic services. International Journal of Nursing Studies, 48(11), 1349-1356.

Care Services Improvement Partnership (CSIP). (2007). A common purpose: Recovery in future mental health services. London: Social Care Institute for Excellence.

Carr, S., \& Harvers, S. (2012). Harnessing hearts and minds for change. In G. Drennan \& D. Aldred (Eds.), Security recovery: Approaches to recovery in forensic mental health settings (Issues in Forensic Psychology) (pp. 115-129). London: Willian Publishing Ltd.

De Vries G. M., Brazil, A. I., Tonkin, M., \& Bulten, H. B. (2016). Ward climate within a high secure forensic psychiatric hospital: Perceptions of patients and nursing staff and the role of patient characteristics. Archives of Psychiatric Nursing, 30(3), 342-349.

Department of Health. (2011). No health without mental health: A cross-government mental health outcomes strategy for people of all ages. London, UK: Department of Health.

Department of Health. (2015). Mental Health Act 1983: Code of Practice. Norwich,UK: The Stationery Office.

Dimond, C., \& Chiweda, D. (2011). Developing a therapeutic model in a secure forensic adolescent unit. Journal of Forensic Psychiatry, 22(2), 283-305.

Drennan, G., \& Aldred, D. (2012). Secure recovery: Approaches to recovery in forensic mental health settings. Oxford, UK: Routledge. 
Finnema, R., Louwerens, J., Slooff, C., \& van-den-Bosch, R. (1996). Expressed emotion on long-stay wards. Journal of Advanced Nursing, 24(3), 473-478.

Hamilton, L. (2010). The boundary seesaw model: Good fences make for good neighbours. In A. Tennant \& K. Howells (Eds.), Using Time, Not Doing Time: Practitioner Perspectives on Personality Disorder and Risk (pp.181-194). London: John Wiley \& Sons.

Hirschi, T. (2002). Causes of Delinquency. New Jersey: Transaction Publishers.

Inglis, A.P. (2010). Therapeutic characteristics of nursing staff in a medium secure setting. Journal of Learning Disabilities and Offending Behaviour, 1(2), 30-46.

Jamieson, E., Taylor, P.J., \& Gibson, B. (2006). From pathological dependence to health independent living: An emergent grounded theory of facilitating independent living. The Grounded Theory Review, 6(1), 79-107.

Joint Commissioning Panel for Mental Health. (2013). Guidance for commissioners of forensic mental health services. [Online] [Accessed on $24^{\text {th }}$ September 2018] https://www.jcpmh.info/resource/guidance-for-commissioners-of-forensicmental-health-services/

Kennedy, G.H. (2002). Therapeutic uses of security: Mapping forensic mental health services by stratifying risk. Advances in Psychiatric Treatment, 8(6), 433-443.

Keski-Valkama, A, Koivisto, M.A., Eronen, M. and Kaltiala-Heino, R. (2010). Forensic and general psychiatric patients' view of seclusion: a comparison study. The Journal of Forensic Psychiatry \& Psychology, 21(3), 446-461.

Livingston, J.D., Rossiter, K.R., \& Verdun-Jones, S.N. (2011). Forensic' labelling: an empirical assessment of its effects on self-stigma for people with severe mental illness. Psychiatry Research, 188(1), 115-122. 
Maguire, T., Young, R., \& Martin, T. (2012). Seclusion reduction in a forensic mental health setting. Journal of Psychiatric and Mental Health Nursing, 19(2), 97-106.

Mann, B., Matias, E., \& Allen, J. (2014). Recovery in forensic services: Facing the challenge. Advances in Psychiatric Treatment, 20(2), 125-131.

Martin, T. and Daffern, M. (2006). Clinician perceptions of personal safety and confidence to manage inpatient aggression in a forensic psychiatric setting. Journal of Psychiatric and Mental Health Nursing, 13(1), 90-99.

Mason, K., and Adler, R. (2012). Group-work therapeutic engagement in a high secure hospital: Male service user perspectives. The British Journal of Forensic Practice, 14(2), 92-103.

McMurran, M. and Ward, T. (2004). Motivating offenders to change in therapy: An organising framework. Legal and Criminology Psychology, 9(2), 295-311.

Meehan, T., McIntosh, W., \& Bergen, H. (2006). Aggressive behaviour in the highsecure forensic setting: The perceptions of patients. Journal of Psychiatric and Mental Health Nursing, 13(1), 19-25.

Mezey, G.C., Kavuma, M., Turton, P., Demetriou, A., \& Wright, C. (2010). Perceptions, experiences and meanings of recovery in forensic psychiatric patients. Journal of Forensic Psychiatry and Psychology, 21(5), 683-696.

Milsom, S., Freesone, M., Duller, R., Bouman, M. and Taylor, C. (2014). Factor structure of the Essen climate evaluation schema measure of social climate in a UK medium-security setting. Criminal Behaviour and Mental Health, 24(2), 8699.

Morris, K., Cox., L.D., \& Ward, K. (2016). Exploring stories of occupational engagement in regional secure unit. The Journal of Forensic Psychiatry \& Psychology, 27(5), 684-697. 
National Survivor User Network (2011). Unlocking service user involvement practice in forensic settings. London: National Service User Network.

Nijdam-Jones, A., Livingston, D.J., Verdun-Jones, S. and Brink, J. (2015). Using social bonding theory to examine 'recovery' in a forensic mental health hospital: A qualitative study. Criminal Behaviour and Mental Health, 25(3), 157-168.

Rae, M.A. (1993). Freedom to Care: Achieving Change in Culture and Nursing Practice in a Mental Health Service. Liverpool: Ashworth Hospital Graphics Department.

Robinson, L. (2007). Balancing rights and risks: Conflicting perspectives in the management of wandering in dementia. Health, Risk \& Society, 9(4), 389-406.

Simpson, A.I.F., \& Penney, S.R. (2011). The recovery paradigm in forensic mental health services. Criminal Behaviour and Mental Health, 21(5), 299-306.

Urheim, R., Rypdal, K., Palmstierna, T., \& Mykletun, A. (2011). Patient autonomy versus risk management: A case study of change in a high security forensic psychiatric ward. International Journal of Forensic Mental Health, 10(1), 41-51.

Ward, T., \& Brown, M. (2004). The good lives model and conceptual issues in offender rehabilitation. Psychology, Crime and Law, 10(3), 243-257.

Whittington, R., \& Richter, D. (2006). From the individual to the interpersonal: Environment and interaction in the escalation of violence in mental health settings. In R. Richter \& Whittington, R (Eds.), Violence in Mental Health Settings: Causes, Consequences, Management (pp. 47-68). New York, NY: Springer. 
Appendix 1: Sample questions used for semi-structured interview

- In your own words, could you please tell me what the term 'least restrictive practice' means to you? (If participant is unable to answer the question, provide definition and information).

- Could you tell me more about your personal experience of 'least restrictive practice' within the hospital that you are in?

- What impact do you think 'least restrictive practices' have had on your care?

- According to the Mental Health Act 1983 'least restrictive practices' are designed to maximise and promote your independence. Thinking about independence, what does independence mean to you?

- How do you think 'least restrictive practices' implemented within your care maximise your independence?

- How do you think these practices could be further improved to maximise and promote your independence?

- According to the Mental Health Act 1983 'least restrictive practices' are also designed to maximise and promote your recovery. What does recovery mean to you?

- How do you think 'least restrictive practices' implemented within your care maximise and promote your recovery?

- How do you think 'least restrictive practices' could be further improved to maximise and promote your recovery? 